\title{
INTERSATELLITE COMMUNICATIONS \\ OPTOELECTRONICS RFSEARCH AT THE \\ GODDARD SPACE FLIGHT CENTER
}

Michael A. Krainak

NASA Goddard Space Flight Center, MC 726.2, Greenbelt, Maryland 20771

\section{$\underline{\text { Abstract }}$}

Current optoelectronics research and development of high power, high bandwidth laser transmitters, high bandwidth, high sensitivity optical receivers, pointing, acquisition and tracking components, and experimental and theoretical system modeling at the NASA Goddard Space Flight Center is reviewed. Program hardware and space flight opportunities are presented.

\section{Introduction}

Free space intersatellite optical communications systems provide a high bandwidth, small size, light weight, low power and low cost alternative to present microwave satellite systems. The first intersatellite optical communications systems will be deployed in space by the US military in the near future. The NASA Goddard Space Flight Center is developing optoelectronics hardware and performing system analysis for the first US civilian experiments.

The present NASA satellite communications system uses microwave technology for space to ground and geosynchronous satellite to low earth orbiting vehicles or platforms. The primary focus of Goddard optical communications researchers is high bandwidth digital repeaters for use between geosynchronous (GEO) satellites.

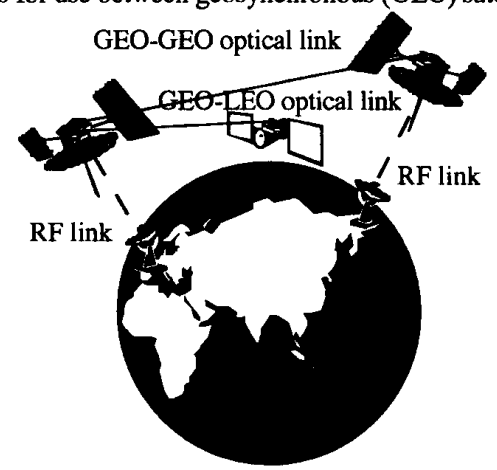

Figure 1. NASA Optical Intersatellite Links Under Research and Development

In this future system, the satellite to ground links would remain in the microwave regime. A secondary effort is the development of optical technology for future low earth orbiting (LEO) space communications users. These links would be from optical terminals on space vehicles or platforms to optical terminals on the TDRSS II system.
The TDRSS satellite repeater system transmits digital data via microwave analog transponders (sometimes referred to as "bent pipes"). On-board processing is needed to provide the interface between the microwave ground-to-space links and the optical space-to-space links thus providing true digita repeaters. The requirements for this interface for the NASA TDRSS system are discussed in references 1 and 2 .

The growth of cable television and the prospects of High Definition Television (HDTV) may provide an opportunity of intersatellite optical communications in the commercial sector. INTELSAT VI is one of the first commercial satellite systems with digital repeaters. 3 The introduction of digital repeater systems reduces the complexity of the microwave-optical interface. This facilitates the introduction of the digital optical intersatellite communications technology.

\section{Laser Transmitter Technology}

The geosynchronous intersatellite optical communication systems under development at NASA requires approximately one watt of optical transmitter laser power ${ }^{4}$. The single channel data rate requirement is 325 megabits per second $(\mathrm{Mb} / \mathrm{s})$. NASA Goddard is investigating two different laser technologies, the aluminum gallium arsenide ( $\mathrm{AlGaAs})$ semiconductor laser diode and the diode pumped Nd doped crystal laser, to meet this requirement. Marshalek et al. ${ }^{5}$ presented a general comparison of these systems.

Commercial fundamental mode $\left(\mathrm{TEM}_{00}\right)$ semiconductor lasers are now available at $150 \mathrm{~mW}$ average power $^{6}$. We have tested the earlier $100 \mathrm{~mW}$ version of these lasers with a $250 \mathrm{Mb} / \mathrm{s}$ Quaternary Pulse Position Modulation (Q-PPM) test signal. An output power of $400 \mathrm{~mW}$ peak $(100$ $\mathrm{mW}$ average) was achieved while meeting the laser pointing, wavefront, and wavelength requirements ${ }^{7}$.

The high power semiconductor laser data rate and output power performance is limited not by laser facet damage, but by the electronic drive circuitry. In order to satisfy the data rate (325 Mbps Q-PPM), optical power (200 $\mathrm{mW}$ peak power from a single diode) and optical to electrical efficiency requirements $(>30 \%)$, a high performance laser current driver is being developed using hybrid circuits ${ }^{8}$. At present, the driver can supply $300 \mathrm{~mA}$ of modulation current with less than a $400 \mathrm{ps}$ rise time. Over $600 \mathrm{~mA}$ of current would be required to operate the $150 \mathrm{~mW}$ average power laser at $600 \mathrm{~mW}$ peak power. The ground based guided wave fiber optic technology has achieved much higher data rates (20 $\mathrm{Gb} / \mathrm{s})^{9}$, but at much lower transmitter optical power ${ }^{10}$.

Laser beamcombiners ${ }^{11}$ have been proposed for achieving the high powers required for intersatellite communications. These systems are cumbersome and have 
been unable to demonstrate long term reliability. An alternative approach to the laser beamcombiner is optical amplification of modulated low power laser light. This has been pursued for many years in continuous wave (CW) arrangements as injection locking ${ }^{12,13}$. When the master laser is modulated with data, this configuration is termed a master oscillator power amplifier (MOPA). We have achieved $340 \mathrm{~mW}$ of diffraction limited CW output power using a broad area laser as the power amplifier. 14 We hope to achieve modulation capability which is only limited by the master oscillator bandwidth.

Certainly the best mechanical stability would come from a fully monolithic structure. Indeed, NASA Goddard is in the process of developing a monolithically integrated Master Oscillator Power Amplifier laser under contract. The near term (1994) goal for this project is to achieve $0.5 \mathrm{~W}$ of CW optical power at modulation bandwidths in excess of 0.5 GHz. Recently, $0.7 \mathrm{~W}$ of $\mathrm{CW}$ power was reported for this laser ${ }^{15}$.

Semiconductor lasers have the advantage of small size, direct modulation capability and high electrical to optical efficiency. However, as noted, the microscopic output aperture and the inability to achieve one watt of optical power without power combining are distinct disadvantages. An alternative is the use of diode lasers as pump sources for a $\mathrm{Nd}$ doped crystal. Multiwatt diffraction limited diode pumped Nd lasers are available and have been space qualified. Unfortunately, no one has demonstrated a high frequency electro-optic modulator which can withstand this high optical power. Multigigahertz Mach-Zehnder waveguide modulators are available for use at 1.06 micron, but have the disadvantages of low optical damage threshold, high RF power requirements and high insertion loss.

One solution to the modulator problem is the use of the master oscillator power amplifier scheme. A high power diode pumped $\mathrm{Nd}$ amplifier can be used to amplify the optical signal from a low power Nd laser modulated by a commercially available electro-optic modulator ${ }^{16}$. A more elegant solution is the use of a diode pumped Nd crystal to amplify the light from a directly current modulated semiconductor laser diode 17 which emits at the $\mathrm{Nd}$ laser wavelength. The Nd laser MOPA (Figure 2) should be able

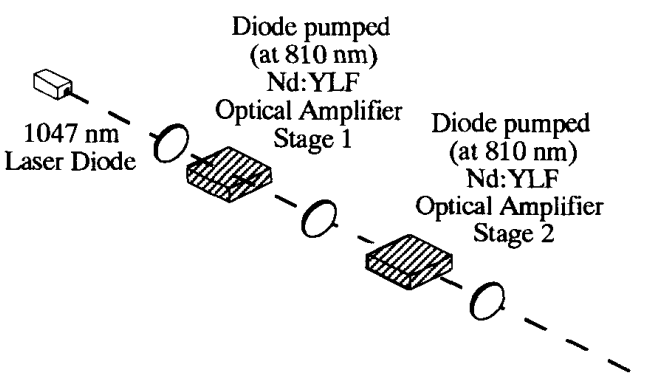

Figure 2. $1047 \mathrm{~nm}$ MOPA Laser Transmitter

to achieve multigigahertz multiwatt operation in the near future. The only disadvantages for this laser result from the 1.06 micron wavelength - larger telescopes and lower receiver sensitivities. Receiver sensitivities are discussed below.

\section{Optical Detector and Receiver Technology}

High power lasers only form one end of an intersatellite optical communications link. High bandwidth, high sensitivity optical receivers are also required. At the AlGaAs semiconductor laser diode wavelength NASA has achieved a receiver sensitivity of 43 photons/bit at a $10^{-6}$ bit error rate at $50 \mathrm{Mb} / \mathrm{s}$ by using Quaternary Pulse Position Modulation (Q-PPM) format and maximum likelihood detection $^{18}$. As a stepping stone to the required $325 \mathrm{Mb} / \mathrm{s}$ data rate, a $220 \mathrm{Mb} / \mathrm{s}$ receiver is under development. Unfortunately, high bandwidth, high sensitivity, avalanche photodiodes and preamplifier combinations are not readily available for use at the $800-860$ or 1.06 micron wavelengths. Impressive results $\left(155\right.$ photons/bit at $10^{-9}$ bit error rate (BER) at $622 \mathrm{Mb} / \mathrm{s}$ ) were achieved, however, at the longer 1.3 micron wavelength in optical waveguides using InGaAs detectors and High Electron Mobility Transistor (HEMT) preamplifiers ${ }^{19}$. Fortunately, it has been shown ${ }^{20}$ that reduced bandwidth receivers can be used at only a minor performance penalty. NASA is developing a low noise avalanche photodiode (APD) and $700 \mathrm{MHz}$ bandwidth preamplifier hybrid integrated circuit via EG\&G Canada using the EG\&G super low ionization $k$-factor (SLIK) APD and a Gigabit Logic preamplifier. Preliminary calculations 21 indicate that this will lead to receiver sensitivities of $\sim 50$ photons/bit at $325 \mathrm{Mb} / \mathrm{s}$ at $10^{-6} \mathrm{BER}$ at the $830 \mathrm{~nm}$ wavelength). This detector preamplifier combination will also be tested at the Nd doped laser wavelengths.

Conventional silicon APD electronic preamplifiers have two disadvantages at the $\mathrm{Nd}$ doped laser wavelengths. The silicon detectors have low quantum efficiency and the low noise preamplifiers have limited bandwidth. We have recently conducted communications experiments at the 1.06 micron wavelength at $50 \mathrm{Mbps}$. A commercial silicon APD enhanced for operation at 900-1100 nm wavelength was used. A sensitivity of 264 photons/bit was achieved at $10^{-6}$ bit error rate. 22 At the $50 \mathrm{Mbps}$ data rate, the $1.06 \mu \mathrm{m}$ systems are very competitive with the $800-860 \mathrm{~nm}$ systems.

As discussed above, one receiver alternative is the use of GaAs detectors and HEMT preamplifiers. Another alternative is optical amplification of the received signal prior to detection. NASA is developing a diode pumped Nd doped fiber preamplifier receiver for this purpose. The front end of the receiver is a commercially available, Nd doped, fiber pumped by a high power semiconductor laser. The detector is either a PIN diode or an APD. Arnaud showed ${ }^{23}$ that this type of receiver has distinct advantages. Telecommunications personnel have achieved receiver sensitivities of 147 photons/bit at $10 \mathrm{~Gb} / \mathrm{s}$ at $10^{-9} \mathrm{BER}$ at the $1.5 \mathrm{micron}$ wavelength with Er doped fiber preamplifiers 24 . This illustrates the multigigahertz bandwidths and high sensitivity available with fiber preamplifier receivers. In a related effort, a monolithic semiconductor optical preamplifier and PIN diode achieved $20 \mathrm{~dB}$ gain and $35 \mathrm{GHz}$ bandwidth 25 .

\section{Acquisition. Pointing and Tracking Detector System Technology}

The development of a novel communications telescope tracking system which eliminates motorized gimbals is being sponsored by NASA. The tracking system operates as follows. A belt driven armature assembly tracks the distant satellite motion near the focal plane of a telescope. An optical relay system on the armature delivers the distant satellite image to the communications detector. The armature is 
attached to a circular disk. Several disks and associated tracking armatures can be stacked allowing the simultaneous tracking of multiple satellites. NASA is developing a 50 Mbps test bed for this Optical Multiple Access communication system. Further details of this system are reported in Reference 26. The proposed application for this technology is communications links from optical terminals on space vehicles and platforms to the TDRSS II system.

\section{Future developments}

The first intersatellite optical communications systems will be deployed in space by the US military in the near future. NASA Goddard continues its development of a ground based $650 \mathrm{Mbps}$ intersatellite optical communications test bed. A space flight operational system using a full flight qualified version of these terminals is the ultimate long-term goal. Unfortunately, at present, this is extremely expensive. A low cost satellite communications experiment can be conducted using these non-space qualified terminals in an evolutionary fashion. The initial communications experiments will be conducted in the laboratory. A satellite communications experiment can be conducted using existing and future satellites with passive reflectors (corner cubes). The Strategic Defense Initiative Relay Mirror Experiment contains several corner cubes, as do the LAGEOS and ERS-1 satellites. A satellite communications link can be established between a ground based single small aperture $(20 \mathrm{~cm})$ transmitter terminal via a satellite corner cube to a ground based large aperture telescope. NASA-Goddard has a 1.2 meter tracking telescope. Initial calculations indicate that at 50 Mbps a few hundred milliwatts of transmitter power at either 820 or $1047 \mathrm{~nm}$ would suffice for a link with a $10^{-6}$ BER on a clear evening. This type of low cost experiment provides an evolutionary path for demonstrating the full capability of intersatellite optical communications.

\section{Acknowledgement}

This paper is a review and summary of the work of many contributors. I would like to acknowledge strong contributions from the following people: D. Cornwell, A Martino, M. Fitzmaurice, G. Unger, W. Hayden and X. Sun

\section{References}

1. "Optical terminal definition for future service growth (FSG) module of ATDRSS". R. Bruno, F. Kalil. AIAA 14th International Communication Satellite systems Conference. March 1992. Paper AIAA-92-1836

2. "RF/Optical interface design for optical intersatellite links". S.J. Campanella and R. K. Garlow. Microwave Journal. 85 1991

3. "INTELSAT VI: The communications system" COMSAT Technical Review. 20 (2) 1990

4. "NASA's system technology development program: a 650 Mbps laser communication testbed." W. L. Hayden, D. Nace, D. Lokerson, P. O. Minott. SPIE Conference 1417 Proceedings. January 1991.

5. "Comparison of optical technologies for intersatellite links in a global telecommunications network." R. G. Marshalek and G. A. Koepf. Optical Engineering 27 (8) 6631988

\section{Spectra Diode Laboratory model 5420 data sheet}

7. "Far-field and phase front characterization of a high power semiconductor laser for free space optical communications".
D. Cornwell, B. Saif. SPIE Conference 1417 Proceedings. January 1991.

8. "High current high bandwidth modulation driver electronics for laser diodes". D. Copeland and R Zimmerman. SPIE Conference 1417 Proceedings. January 1991.

9. "20 Gbit/s soliton transmission over $200 \mathrm{~km}$ using erbiumdoped fibre repeaters". Electronics Letters 26 (19) 15921990

10. "10 Gbit/s optical transmitter module with MQW DFB-LD and DMT driver IC". T. Suzaki, Y. Suzaki, H. Yamada, S. Fujita, H. Hida, M. Kitamura and M. Shikada. Electronics Letters 26 (2) 1511990

11. "Selection of laser diode beam combining techniques for free space communication." D. L. Begley, W. L. Casey, K. O. Lippold. SPIE Proceedings 610 276. 1986

12. "Injection locking of coupled-stripe diode laser arrays". L. Goldberg, H. F. Taylor, J. F. Weller and D. R. Scifres. Applied Physics Letters 46 236. February 1985.

13. "Modeling of injection locking phenomena in diode laser arrays." Optics Letters 11 144. March 1986.

14. "Modulation Characteristics of a Semiconductor Master Oscillator-Broad-Area Power Amplifier (MOPA) Configuration". Donald M. Cornwell, Jr. CLEO 1992 Paper CThI12

15. "High-power diffraction-limited, monolithically integrated master oscillator/ power amplifier". D. F. Welch, R. G. Waarts, D. G. Mehuys, R. Parke, D. R. Scifres, R. R. Craig, W. S. Streifer. SPIE Conference 1418 Proceedings. January 1991.

16. "Diode pumped Nd:YAG laser transmitter for free-space optical communications. E. Nava, G. Re Garbagnati, M. Garbi, L. D. Marchiori, A. E. Marini." SPIE Conference 1417 Proceedings. January 1991.

17. "High power $1.06 \mu \mathrm{m}$ GaInAsP DCPBH lasers". T. Van Dongen et al. Electronics Letters 25 (19) 12771989

18. " $50 \mathrm{Mbps}$ free space direct detection laser diode optical communication system with $Q=4$ PPM signaling". $X$. Sun F. Davidson and C. Field. SPIE 12183851990

19. "A high-performance optical receiver for $622 \mathrm{Mb} / \mathrm{s}$ directdetection systems". L. Tzeng, R. Frahm and W. Asous. IEEE Photonics Technology Letters 2 (10) 759 October 1990.

20."Bandwidth requirements for direct detection optical communication receivers with PPM signaling." X. Sun, F. Davidson, M. Krainak. SPIE Conference 1417 Proceedings. January 1991

21. "Numerical simulation of a $325 \mathrm{Mbit} / \mathrm{s}$ QPPM Optical Communication System." A. Martino. SPIE Conference Proceedings. January 1992.

22. "High sensitivity $1064 \mathrm{~nm} 50$ Mbps direct detection free space communications receiver". M. Krainak, G. Unger and X. Sun. CLEO Technical Digest. May 1992. Paper CTHI16.

23. "Enhancement of optical receiver sensitivities by amplification of the carrier." J. A. Arnaud. IEEE Journal of Quantum Electronics QE-4 (11) 1968. 
24. "High receiver sensitivity at $10 \mathrm{~Gb} / \mathrm{s}$ using an Er-doped fiber preamplifier pumped with a $0.98 \mu \mathrm{m}$ laser diode". T. Saito et al. IEEE Photonics Technology Letters 3 (6) 551 1991.

25. "Monolithic integration of a $1.5 \mu \mathrm{m}$ optical preamplifier and PIN photodetector with a gain of $20 \mathrm{~dB}$ and a bandwidth of $35 \mathrm{GHz}^{\circ}$. D. Wake, S. N. Judge, T. P. Spooner, M. J. Harlow, W. J. Duncan, I. D. Henning and M. J. O'Mahony. Electronics Letters 26 (15) 11661990

26. "GSFC conceptual design study for an intersatellite optical multiple access communication system". N. Fox, E. Clarke, W. Maynard and R. Bruno . SPIE Conference 1417 Proceedings. January 1991. 